\title{
Função lútea em adolescentes normais com ciclos menstruais regulares
}

\author{
Luteal function in normal adolescents with regular menstrual cycles
}

\author{
Zuleide Aparecida Felix Cabral ${ }^{1}$, Laudelino de Oliveira Ramos ${ }^{2}$, \\ Angela Maggio da Fonseca ${ }^{3}$, Sebastião Freitas de Medeiros ${ }^{4}$
}

\section{RESUMO}

Objetivo: examinar a função lútea em adolescentes com ciclos menstruais regulares. Métodos: foram incluídas neste estudo, de coorte prospectivo, 55 adolescentes eumenorrêicas, com idade entre 14 e 19 anos e menarca aos 12,2 anos. Examinaram-se o dia da ocorrência de ovulação, o grau de vascularização do corpo lúteo e o índice de resistência dos vasos ovarianos, as concentrações de progesterona e a resposta do endométrio. A ovulação foi identificada por ultra-sonografia transvaginal a partir do $2^{\circ}$ ou $5^{\circ}$ dia do ciclo, repetindo-a a cada dois dias. A vascularização e o índice de resistência lútea foram aferidos por dopplervelocimetria no $10^{\circ}$ dia pós-ovulação. Os níveis de progesterona sérica foram estimados por quimioluminescência nos dias 6, 9 e 12 da fase lútea. A histologia endometrial foi analisada após biópsia feita entre o $8^{\circ}$ e o $10^{\circ}$ dia pós-ovulação. Usouse software SPSS para análise estatística, assumindo-se significância quando $p<0,05$. Resultados: A ovulação foi observada em média no $17^{\circ}$ dia. Os níveis séricos de progesterona foram de 11,4, 10,9 e 3,9 ng/mL respectivamente nos dias 6, 9 e 12 pósovulação; a média dos níveis de progesterona nos três dias foi de 10,3 ng/mL. A vascularização do corpo lúteo foi escassa em $34,6 \%$, moderada em $23,6 \%$ e exuberante em $41,8 \%$. O índice de resistência foi de 0,441 dez dias após a ovulação. O endométrio mostrou-se sincrônico em 85,5\% das biópsias e fora de fase em 14,5\%. As correlações entre o dia da ovulação e o tipo de endométrio $(\mathrm{p}=0,29)$, níveis de progesterona e o tipo endométrio $(\mathrm{p}=0,454)$, grau de vascularização $(\mathrm{p}=0,99)$ e índice de resistência do corpo lúteo $(\mathrm{p}=0,23)$ não foram estatisticamente significantes. A histologia endometrial e o grau de vascularização também não mostraram associação $(\mathrm{p}=0,61)$. Conclusão: a função lútea em adolescentes eumenorrêicas, avaliada pela datação do endométrio em ciclo único, mostrou-se anormal em 14,5\%. As variáveis grau e intensidade da vascularização, concentração sérica de progesterona ou dia da ocorrência de ovulação não mostraram associação com a histologia endometrial.

PALAVRAS-CHAVE: Fase luteal/fisiologia; Ciclo menstrual; Adolescente; Estudos retrospectivos; Estudos de coortes

\section{ABSTRACT}

Purpose: to evaluate the luteal function in adolescents with regular menstrual cycles. Methods: this prospective cohort study included 55 adolescents, aged 14-19 years, with menarche at 12.2 years. Ovulation was identified by ultrasound, starting on the second or fifth day of the cycle. The corpus luteum vascularization and the resistence index of the ovarian vessels were measured by Doppler on the tenth postovulatory day. Progesterone was measured by chemoluminescence on days 6,9 and 12 of the luteal phase. The endometrial biopsy was performed 8 to 10 days after ovulation. The results were analyzed using the SPSS software and were considered significant when $p<0.05$. Results: on average ovulation was on day 17. Progesterone levels were $11.4,10.9$ and $3.9 \mathrm{ng} / \mathrm{mL}$ on days 6,9 , and 12 after ovulation, respectively; the progesterone mean during the whole luteal phase was $10.3 \mathrm{ng} / \mathrm{mL}$. Luteal vascularization was scarce in $34.6 \%$, mild in $23.6 \%$ and exuberant in $41.8 \%$. The resistance index was 0.441 . On the tenth day post-ovulation the endometrium was normal in $85.5 \%$ and outof-phase in $14.5 \%$. There was no correlation between the ovulation day and endometrial dating ( $p=0.294)$, levels of progesterone and endometrial dating $(\mathrm{p}=0.454)$, progesterone and corpus luteum vascularization $(\mathrm{p}=0.994)$, or resistance index $(\mathrm{p}=0.237)$. There also was no association between endometrium development and degree of vascularization $(\mathrm{p}=0.611)$. Conclusion: abnormal luteal function in adolescents with regular menstrual cycles was found in $14.5 \%$. Degree of vascularization, resistance index, and serum progesterone were not related to endometrium development.

KEYWORDS: Lutheal fase/physiology; Menstrual cycle; Adolescent; Retrospective studies; Cohort studies

1 Professora Adjunta do Departamento de Ginecologia e Obstetrícia da Faculdade de Ciências Médicas da Universidade Federal de Mato Grosso - UFMT - Cuiabá (MT) - Brasil - Hospital Universitário Julio Muller.

2 Professor Associado Livre Docente do Departamento de Obstetrícia e Ginecologia Faculdade de Medicina, Universidade de São Paulo - USP - São Paulo (SP) - Brasil.

3 Professora Associada Livre Docente do Departamento de Obstetrícia e Ginecologia Faculdade de Medicina, Universidade de São Paulo - USP - São Paulo (SP) Brasil.

4 Professor Adjunto do Departamento de Ginecologia e Obstetrícia da Faculdade de Ciências Médicas, Universidade Federal de Mato Grosso - UFMT Cuiabá (MT) - Brasil - Hospital Universitário Julio Muller.

Correspondência: Zuleide Aparecida Felix Cabral

Rua M n. 45, Edifício Manchester - Bairro Miguel Sutil - 78048- 230 Cuiabá - MT - Telefone - Fax: 0XX (65) - 3624 5989 - email: hzcabral@terra.com.br 


\section{Introdução}

A menarca, mesmo considerada excelente marcador clínico da maturação ovariana, não comprova que a maturidade sexual e a capacidade reprodutiva estejam completamente estabelecidas. Após intervalo de meses, ou anos, seguindo a menarca, as ovulações tornam-se regulares, com normalização dos ciclos menstruais. O estabelecimento de ciclos ovulatórios normais indica que o desenvolvimento dos mecanismos envolvidos para a sua ocorrência está completo. Na adolescência é possivel a coexistência de insuficiência folicular e luteínica, podendo ainda ocorrer ciclos menstruais ovulatórios intercalados com ciclos anovulatórios, resultando em diferentes formas de sangramento menstrual ${ }^{1,2}$.

Vários métodos e indicadores clínicos têm sido utilizados para a caracterização da fase folicular, ovulação e função lútea. Dá-se maior importância à ultra-sonografia, dopplervelocimetria, biópsia do endométrio e dosagem das gonadotrofinas hipofisárias, estradiol e progesterona. No entanto, a precisão no diagnóstico do dia da ocorrência da ovulação permanece limitada e os complexos mecanismos envolvidos nas disfunções das fases folicular ou lútea ainda não estão completamente entendidos. Para melhor precisão diagnóstica das disfunções das fases folicular ou lútea, recomenda-se a associação de vários métodos propedêuticos ${ }^{3}$. A insuficiência da fase lútea, considerada uma das mais enigmáticas e sutis anormalidades ovulatórias, caracteriza-se pela ocorrência de ovulação com luteólise precoce e/ ou produção deficiente de progesterona pelo corpo lúteo, dificultando a concepção e/ou manutenção da gravidez.

A identificação de ovulação e a avaliação da função lútea na adolescência têm recebido pouca atenção dos fisiologistas e clínicos e por esse motivo há escassez de informações epidemiológicas e clínicas acerca do padrão das fases do ciclo menstrual em adolescentes eumenorrêicas ${ }^{4}$. No entanto, a correta avaliação das fases folicular e lútea é relevante em reprodução, na avaliação de casais inférteis, independente da idade da mulher. Os estudos ora disponiveis foram realizados em mulheres de outra faixa etária. Mesmo que muitos mesclem, em suas casuísticas, adolescentes menstruando regularmente com idade entre $18 \mathrm{e}$ 19 anos, estes trabalhos foram realizados para a investigação de infertilidade e não em mulheres clinicamente normais. A falta de informações sobre a função lútea em adolescentes normais, com ciclos menstruais regulares, combinada com a variação na estimativa de sua prevalência, indefinição dos métodos propedêuticos utilizados na sua avaliação e a discutivel importância clínica das suas alterações em mulheres não adolescentes $^{5,6}$, motivaram a realização deste estudo.

\section{Métodos}

Foram incluídas no estudo 55 adolescentes que procuraram o Ambulatório de Ginecologia Infanto-Juvenil do Departamento de Ginecologia e Obstetrícia da Faculdade de Ciências Médicas da Universidade Federal de Mato Grosso (UFMT), Hospital Universitário Julio Muller (HUJM), procurando orientação para o uso de anticoncepção. A idade variou de 14 a 19 anos (mediana 16,9 $\pm 1,4$ anos). A menarca ocorreu entre 9 e 14 anos (média de 12,2 $\pm 1,2$ anos). Como critério de inclusão admitiram-se apenas adolescentes que apresentavam periodo de tempo após a menarca superior ou igual a doze meses, mantinham atividade sexual e tinham tido pelo menos três ciclos menstruais regulares, consecutivos, antes do início do estudo. Consideraram-se como regulares os ciclos menstruais com intervalo entre vinte e cinco e trinta e cinco dias ${ }^{1}$. Estabeleceram-se como critérios de exclusão adolescentes tabagistas, praticantes de esportes extenuantes, portadoras de doença neoplásica, lactantes ou já usuárias de anticoncepção hormonal.

Todas as adolescentes foram avaliadas durante todo um ciclo menstrual, antes de iniciarem o método de contracepção escolhido. O estudo foi aprovado pelo Comitê de Ética em Pesquisa do Hospital Universitário Júlio Muller da Faculdade de Ciências Médicas da Universidade Federal de Mato Grosso e pela Comissão de Ética para Análise de Projetos de Pesquisa da Diretoria Clínica do Hospital das Clínicas da Faculdade de Medicina da Universidade de São Paulo. O termo de consentimento livre esclarecido foi assinado por todas as adolescentes e seus responsáveis. Este estudo, de coorte prospectivo, propôs examinar o dia da ocorrência de ovulação, o grau de vascularização do corpo lúteo e o índice de resistência destes vasos, os níveis séricos de progesterona na fase lútea e a resposta do estroma e glândulas endometriais aos esteróides ovarianos.

O monitoramento da fase folicular pela ultrasonografia transvaginal foi feito com aparelhos Toshiba Eccocee e ATIL HDI 9 com transdutor endocavitário de 5 a $7 \mathrm{MHz}$. Os exames foram realizados entre o segundo e o quinto dia do ciclo menstrual e, posteriormente, a cada dois dias, até 
o aparecimento de sinais de rotura folicular. Considerou-se que houve ovulação quando a imagem folicular desapareceu e/ou evidenciaram-se imagens sugestivas de corpo lúteo: colapso folicular, identificação de estrutura hipoecogênica com parede irregular ou aparecimento de estrutura sólida ou complexa. O dia da ovulação foi considerado como o primeiro dia da fase lútea. Todas as adolescentes tiveram o ciclo menstrual totalmente avaliado pelo mesmo ultra-sonografista.

No décimo dia pós-ovulação avaliaram-se a vascularização e o índice de resistência do corpo lúteo com dopplervelocimetria. O grau de vascularização foi classificado de forma subjetiva, de acordo com a porcentagem de distribuição do fluxo sanguíneo ao redor do corpo lúteo. Considerou-se como vascularização escassa a presença de fluxo sangüíneo circundando menos de 30\% da área do corpo lúteo, vascularização moderada quando o fluxo sangüíneo circundou o corpo lúteo entre 30 a $70 \%$ da sua área e vascularização exuberante na presença de fluxo sangüíneo circundando mais de $70 \%$ da área do corpo lúteo. O índice de resistência do corpo lúteo foi avaliado por meio do estudo dos vasos intra-ovarianos e calculado automaticamente subtraindo-se a velocidade sistólica máxima da velocidade diastólica e dividindo-se o resultado pela velocidade sistólica (IR $=\mathrm{vs}-\mathrm{vd} / \mathrm{vs})^{7}$.

O sangue para a determinação do nível sérico de progesterona foi colhido no sexto, nono e décimo segundo dia após a ovulação. O soro foi separado do sangue total e estocado à temperatura de $-20^{\circ} \mathrm{C}$. As amostras foram dosadas, em duplicata, por quimioluminescência de fase sólida, utilizandose o teste Immulite Progesterone I (DPCDiagnostic Products Corporation, Los Angeles, USA). Todas as amostras de uma mesma adolescente foram dosadas no mesmo ensaio, sendo calculada a média dos dois valores obtidos. Os resultados foram expressos em $\mathrm{ng} / \mathrm{mL}$. A concentração mínima detectável neste método é de $0,2 \mathrm{ng} / \mathrm{mL}$ e o limite do ensaio é de $40 \mathrm{ng} / \mathrm{mL}$. Os coeficientes de variação intra-ensaio foram de $8,8,5,4$ e $6,1 \%$ para as concentrações de $1,4,3,1$ e $13,3 \mathrm{ng} / \mathrm{mL}$, respectivamente. As variações interensaio foram de $8,1,6,0$ e $4,8 \%$ para as concentrações de 1,4, 3,2 e 13,4 ng/mL, respectivamente.

Entre o oitavo e o décimo dia após a ovulação, as adolescentes foram submetidas à biopsia do endométrio. O material foi obtido da parede anterior média uterina, área de desenvolvimento endometrial máximo ${ }^{8}$, utilizando-se cureta de Novak modificada. O material foi colocado em frascos contendo solução de formol e, posteriormente, incluso em parafina e corado com hematoxilinaeosina. A datação do endométrio incluiu os oito aspectos histológicos descritos por Noyes: mitose glandular, pseudo-estratificação nuclear, vascularização basal, secreção glandular, edema estromal, reação pseudodecidual, mitose estromal e infiltração leucocitária ${ }^{9}$.

Para análise estatística utilizou-se o software SPSS (Statistical Package for the Social Sciences) 11.0. Assumindo prevalência de insuficiência lútea de $3,7 \%{ }^{10}$, erro de $5 \%$ na estimativa e intervalo de confiança de $95 \%$, a amostra necessária foi de 55 indivíduos ${ }^{11}$. A duração do ciclo, definido como o número de dias entre o primeiro dia da menstruação e o dia que antecedeu o próximo fluxo menstrual, exibiu distribuição simétrica e foi expressa como média e erro padrão da média. O dia da ovulação, definido pela ultrasonografia, mostrou distribuição assimétrica (teste de Liliefors) e presença de um outlier (valor discrepante e muito distante dos demais) e foi expresso como mediana e intervalo de confiança de 95\%. As concentrações de progesterona no sexto, nono e décimo segundo dia também tiveram distribuição assimétrica e foram expressas como mediana e IC 95\%. Identificou-se a existência de um outlier no dia 6 e outro no dia 9 pós-ovulação (teste de Grubbs), sendo ambos excluídos dos cálculos. As variáveis indice de resistência da vascularização lútea e concentração média de progesterona em todo o ciclo mostraram distribuição normal, sendo descritas como média e erro padrão da média $(\overline{\mathrm{x}} \pm \mathrm{EPM})$. As variáveis qualitativas, grau de vascularização do corpo lúteo e compatibilidade do endométrio com o dia do ciclo padronizado foram expressas em proporções com intervalos de confiança de 95\% (método de Wald). Fez-se a comparação das medianas dos níveis de progesterona nos dias 6, 9 e 12 após a ovulação por análise de variância de um critério (Kruskal-Wallis), seguida do teste de comparação múltipla de Tukey.

A verificação de associação entre as médias de concentração de progesterona e o índice de resistência dos vasos lúteos foi feita por regressão linear, expressa pelo coeficiente de correlação de Pearson (r) e analisada pelo teste $t$ de Student para amostras não pareadas. A comparação da duração do ciclo ou dia da ovulação entre os grupos com endométrio concordante ou discordante foi feita pelo teste $t$ de Student. Examinou-se a associação entre indivíduos com níveis séricos de progesterona maior ou menor que $10 \mathrm{ng} / \mathrm{mL}$ ou grau de vascularização do corpo lúteo com o tipo de endométrio pelo teste exato de Fisher. A análise de possivel associação entre os resultados da biópsia com o grau de vascularização do corpo lúteo foi examinada pelo teste do $\chi^{2}$. 


\section{Resultados}

A ovulação, identificada pela ultrasonografia, ocorreu entre o $12^{\circ}$ e o $28^{\circ}$ dia do ciclo menstrual, mediana de 17 dias (IC $95 \%, 15$ a 17). As concentrações de progesterona no sexto, nono e décimo segundo dia da fase lútea foram, respectivamente, $11,4 \mathrm{ng} / \mathrm{mL}$ (IC 95\%: 10,1-12,8), 10,9 $\mathrm{ng} / \mathrm{mL}$ (IC 95\%: 9,6-12,7) e 3,9 ng/mL (IC 95\%: 2,2-6,2) e a média geral da concentração de progesterona na fase lútea obtida pela inclusão de todos os valores obtidos no sexto, nono e décimo segundo dia foi de 10,3 $\pm 0,7 \mathrm{ng} / \mathrm{mL}$. À dopplervelocimetria, vascularização escassa do corpo lúteo foi evidenciada em 19 adolescentes (34,6\%; IC 95\%: 23,3-47,8), moderada em 13 (23,6\%; IC 95\%: 14,3$36,5)$ e exuberante em 23 (41,8\%; IC 95\%: 29,7$55,0)$. O índice de resistência vascular, verificado no $10^{\circ}$ dia da fase lútea, foi de $0,44 \pm 0,01$. A diferenciação do endométrio mostrou-se sincrônica em 85,5\%, (IC 95\%: 69,5-90,0) das adolescentes e com atraso superior a dois dias em 14,5\% (IC 95\%: $7,3-26,5)$. As concentrações de progesterona mantiveram-se estáveis entre o sexto e o nono dia pósovulação, mas significativamente inferior no décimo segundo dia $(\mathrm{p}<0,0001)$, declinando em cerca de $65,3 \%$ (IC 95\% 38,5-88,6) já no décimo segundo dia. Para maior clareza os resultados estão resumidos na Tabela 1 .

Tabela 1 - Parâmetros clínico-laboratoriais da função lútea em adolescentes com ciclos menstruais regulares

\begin{tabular}{lccc}
\hline Parâmetro & Média/Mediana & EPM & IC \\
\hline Intervalo do ciclo menstrual (dias) & 29,5 & 0,22 & $29,1-30,0$ \\
Dia da ovulação & 17,0 & & $15-17$ \\
Duração da fase lútea (dias) & 13,0 & & $12-14$ \\
Concentração de progesterona (ng/mL) & 10,3 & 0,71 & $8,9-11,8$ \\
P, dia 6 da fase lútea & 11,4 & & $10,1-12,8$ \\
P, dia 9 da fase lútea & 10,9 & & $9,6-12,7$ \\
P, dia 12 da fase lútea & 3,9 & & $2,2-6,2$ \\
IR & 0,44 & 0,01 & $0,41-0,47$ \\
\hline
\end{tabular}

$\mathrm{P}=$ progesterona, $\mathrm{EPM}=$ erro padrão da média, $\mathrm{IC}=$ intervalo de confiança, $\mathrm{IR}=$ índice de resistência dos vasos intra-ovarianos.

O índice de resistência da vascularização lútea não mostrou estar associado às concentrações de progesterona $(r=0,16 ; p=0,23)$. A duração do ciclo menstrual entre adolescentes cuja biópsia mostrou endométrio compativel e as adolescentes com diferenciação endometrial atrasada não demonstrou diferença significante $(r=0,16$; $\mathrm{p}=0,11)$. Do mesmo modo, não houve diferença significante entre o dia de ocorrência de ovulação e o tipo do endométrio $(\mathrm{r}=0,07 ; \mathrm{p}=0,29)$. Não se de- monstrou haver associação entre o tipo de endométrio com as concentrações de progesterona superiores $(\mathrm{p}=0,45)$ ou inferiores a $10 \mathrm{ng} / \mathrm{mL}$ $(p=0,41)$. Não se demonstrou também associação entre tipo de endométrio e o grau de vascularização lútea $(p=0,61)$ ou concentrações médias de progesterona nos dias 6,9 e 12 da fase lútea com a intensidade da vascularização do corpo lúteo $(p=0,99)$. Em conjunto, as associações examinadas são mostradas na Tabela 2 .

Tabela 2 - Correlação entre variáveis clínico-laboratoriais utilizadas na avaliação da função lútea em adolescentes com ciclos menstruais regulares

\begin{tabular}{lcc}
\hline Associação testada & $\begin{array}{c}\text { Correlaçãol } \\
\text { Associação }\end{array}$ & Valor $p$ \\
\hline Duração do ciclo menstrual vs tipo de endométrio & 0,168 & $p=0,117^{*}$ \\
Dia da ovulação vs tipo de endométrio & 0,075 & $p=0,294^{*}$ \\
Níveis de P vs tipo de endométrio & 0,016 & $p=0,454^{* *}$ \\
Grau de vascularização vs tipo de endométrio & 0,132 & $p=0,611^{* *}$ \\
Níveis de P vs grau de vascularização do CL & 0,038 & $p=0,994^{* * *}$ \\
Níveis de P vs índice de resistência do CL & 0,166 & $p=0,237^{*}$ \\
\hline$P=$ progesterona, $C L=$ corpo lúteo. & & \\
${ }^{*}$ teste $t,{ }^{* *}$ teste de Fisher, ${ }^{* * * t e s t e ~ d o ~} \chi^{2}$. & &
\end{tabular}

\section{Discussão}

$\mathrm{Na}$ adolescência, níveis elevados de progesterona sérica têm sido utilizados como prova indireta da existência de ovulação após a menarca. Em mulheres adultas a avaliação dos niveis de progesterona na fase lútea é utilizada no diagnóstico da ovulação e avaliação da função do corpo lúteo ${ }^{3}$. Ainda que seja método menos invasivo, a definição da concentração de progesterona que discrimina a atividade lútea como adequada ou inadequada permanece controversa ${ }^{12}$. Além da sobreposição de valores entre mulheres normais e mulheres com fase lútea deficiente, o fato de a secreção de progesterona ser pulsátil torna a interpretação mais difícil ${ }^{13}$. Têm sido propostas várias coletas sanguíneas e diferentes pontos de corte para discriminar função lútea normal e anormal. Valor superior a este limite seria encontrado em ciclos normais e inferior em mulheres com insuficiência lútea. Niveis de progesterona plasmática superiores a 3 ou $10 \mathrm{ng} / \mathrm{mL}$ seriam indicativos de função lútea normal ${ }^{12,14}$. O critério de três dosagens seriadas antes da menstruação, somando entre 15 e $30 \mathrm{ng} / \mathrm{mL}$, caracterizaria fase lútea normal ${ }^{15}$.

A avaliação da concentração plasmática de progesterona realizada no presente estudo em três dias diferentes mostrou que somente três adoles- 
centes $(5,4 \%)$ tiveram concentração de progesterona inferior a $3 \mathrm{ng} / \mathrm{mL}$ em dosagem isolada, após a ovulação ser demonstrada pela ultra-sonografia. No entanto, quando se considerou a média das três dosagens, o resultado foi superior a $10 \mathrm{ng} / \mathrm{mL} \mathrm{em}$ todas as adolescentes. Apesar de níveis de progesterona superiores a $3 \mathrm{ng} / \mathrm{mL}$, nem todos os casos apresentam maturação histológica do endométrio compativel com a fase lútea à biopsia, sugerindo que niveis de progesterona e datação do endométrio não mostram necessariamente boa associação para avaliação da função lútea. Entretanto, boa correlação entre essas duas variáveis foi observada recentemente em pequena amostra de onze mulheres férteis, com idade superior a 18 anos, tendo o dia da ovulação fundamentado na dosagem urinária do hormônio luteinizante ${ }^{16}$.

As concentrações de progesterona observadas no presente estudo foram superiores na fase lútea precoce e inferiores na fase lútea média, quando comparadas às descritas anteriormente com o uso de radioimunoensaio ${ }^{13}$. A diferença encontrada entre estes estudos parece não ser explicada apenas por diferenças na sensibilidade ou especificidade entre a quimioluminescência e o radioimunoensaio. Resultados conflitantes podem estar relacionados ainda com a concentração de componentes capazes de interferir com um determinado ensaio. Tanto pela inexistência de informações como por maior clareza, estudos comparando os niveis de progesterona na fase lútea com os dois métodos são ainda necessários, principalmente quando muitos laboratórios seguem usando radioisótopos. A redução de $65 \%$ observada nos níveis de progesterona já no décimo segundo dia após a ovulação sugere que a avaliação do nivel de progesterona, quando solicitada em dosagem única, deva ser mais precoce. A quantificação de progesterona, além de gonadotrofinas e estradiol, é essencial para definir fase folicular e luteínica adequadas em ciclos menstruais ovulatórios ${ }^{17}$.

A biópsia do endométrio, utilizada como bioensaio de progesterona produzida pelo corpo lúteo, examinou possível sincronia entre o desenvolvimento do tecido glandular/estroma do endométrio com o dia da fase lútea ${ }^{18}$. Todos os endométrios avaliados foram secretores, com características histológicas de ciclos menstruais ovulatórios. Houve, assim, absoluta concordância com a ultra-sonografia no diagnóstico de ovulação. A grande porcentagem de endométrios cronologicamente compativeis encontrada neste estudo pode ser justificada por ser a amostra estudada representada por jovens eumenorrêicas, todas com ovulação constatada pela ultra-sonografia e con- centração média de progesterona igual ou superior $10,0 \mathrm{ng} / \mathrm{mL}$, em três dosagens seriadas.

Os estudos nos quais se avalia tanto a morfologia como a capacidade funcional do ovário, por meio da associação da ultra-sonografia transvaginal com dopplervelocimetria, mostram resultados divergentes ${ }^{19,20}$. Possivelmente, as atuais divergências sejam resultantes das distintas características populacionais, tipo de vaso avaliado, tipo de Doppler empregado e critérios usados na quantificação da vascularização ovariana ${ }^{21}$. Os índices de resistência do corpo lúteo encontrados no presente estudo são semelhantes aos observados tanto em mulheres férteis como em mulheres com fase lútea anormal ${ }^{7}$. Não se encontrou correlação entre as concentrações de progesterona com vascularização ou índice de resistência do corpo lúteo. Resultados semelhantes foram descritos anteriormente tanto entre mulheres férteis como entre mulheres com fase lútea deficiente ${ }^{22,23}$. É certo que outros fatores, além da concentração de progesterona, podem estar envolvidos na dinâmica vascular e função ovariana ${ }^{24,25}$. É questionável, no entanto, se a intensidade de vascularização ou indice de resistência dos vasos ovarianos possam estar relacionados com a função do corpo lúteo.

Os ciclos menstruais referidos pela maioria das adolescentes aqui avaliadas variaram de 28 a 30 dias, mas a simples presença de ciclos menstruais com intervalos regulares parece não ser parâmetro clínico seguro para o diagnóstico de ovulação. Há estudos clínicos relatando a presença de anovulação em ciclos menstruais regulares e ovulação em ciclos menstruais irregulares ${ }^{26}$. A associação dos parâmetros regularidade menstrual e ultra-sonografia torna o diagnóstico de ovulação mais preciso. A duração do ciclo menstrual também parece não ser critério seguro para avaliação da função fase lútea, mas fase lútea deficiente raramente é encontrada em mulheres com ciclos menstruais com intervalos entre vinte e oito e trinta dias. Parece que mais de 95\% das mulheres que apresentam ciclos menstruais regulares têm concentração plasmática de progesterona e biópsia endometrial compatíveis com ovulação. Por outro lado, aproximadamente $75 \%$ das mulheres com irregularidade menstrual apresentam concentração plasmática de progesterona em níveis considerados normais. Portanto, ciclos menstruais irregulares não significam anovulação, mas podem ser indicadores da necessidade de avaliação hormonal mais minuciosa ${ }^{27}$. Este estudo avaliou a fase lútea em adolescentes com ciclos menstruais regulares, permitindo evidenciar características peculiares desta população. Os achados sugerem que a regularidade menstrual em ado- 
lescentes parece ser indicador de função lútea adequada.

Ainda que esta investigação seja do tipo coorte prospectivo e inclua amostra de tamanho adequado, outros estudos, estimando a avaliação por mais de um ciclo e incluindo novas variáveis, serão importantes para testar os resultados desta presente pesquisa.

\section{Referências}

1. Creinin MD, Keverline S, Meyn LA. How regular is regular? An analysis of menstrual cycle regularity. Contraception. 2004;70(4):289-92.

2. Harel Z. Approach to the adolescent girl as she transits from irregular to regular menstrual cycles. J Pediatr Adolesc Gynecol. 2005;18(2):193-200.

3. Crosignani PG, Rubin BL. Optimal use of infertility diagnostic test and treatments. The ESHRE Capri Workshop Group. Hum Reprod. 2000;15(3):723-32.

4. Jarvelaid M. The effect of gynecologic age, body mass index and psychosocial environment on menstrual regularity among teenaged females. Acta Obstet Gynecol Scand. 2005;84(7):645-9.

5. Olive DL. The prevalence and epidemiology of lutealphase deficiency in normal and infertile women. Clin Obstet Gynecol. 1991;34(1):157-66.

6. Bukulmez O, Arici A. Luteal phase defect: myth or reality. Obstet Clin North Am. 2004;31(4):727-44.

7. Kupesic S, Kurjak A. The assessment of normal and abnormal luteal function by transvaginal color Doppler sonography. Eur J Obstet Gynecol Reprod Biol. 1997;72(1):83-7.

8. Davis OK, Berkeley AS, Naus GJ, Cholst IN, Freedman KS. The incidence of luteal phase defect in normal, fertile women, determined by serial endometrial biopsies. Fertil Steril. 1989;51(4):582-6.

9. Noyes RW, Hertig AT, Rock J. Dating the endometrial biopsy. Fertil Steril. 1950;1(1):3-25.

10.Jones GS. The luteal phase defect: a review of pathophysiology. Curr Opin Obstet Gynecol. 1991;3(5):641-8.

11. Daly LE, Bourke GJ. Sample size determination. In: Daly LE, Bourke GJ, McGilvray J. Interpretation and uses of medical statistics. 5th ed. Oxford: Blackwell Sciences; 2000. p. 269-95.

12.Li TC, Cooke ID. Evaluation of the luteal phase. Hum Reprod. 1991;6(4):484-99.

13. Filicori M, Butler JP, Crowley WF Jr. Neuroendocrine regulation of the corpus luteum in the human. Evidence for pulsatile progesterone secretion. J Clin Invest. 1984;73(6):1638-47.
14. Daya S, Ward S. Diagnostic test properties of serum progesterone in the evaluation of luteal phase defects. Fertil Steril. 1988;49(1):168-70.

15. Hinney B, Henze C, Kunh W, Wuttke W. The corpus luteum insufficiency: a multifactorial disease. J Clin Endocrinol Metab. 1996;81(2):565-70.

16. Santoro N, Goldsmith LT, Heller D, Illsley N, McGovern P, Molina C, et al. Luteal progesterone relates to histological endometrial maturation in fertile women. J Clin Endocrinol Metab. 2000;85(11):4207-11.

17. Nakajima ST, Molly MH, Oi RH, Ohlson KA, Azevedo RA, Boyers SP. Clinical evaluation of luteal function. Obstet Gynecol. 1994;84(2):219-21.

18. Fadare $\mathrm{O}$, Zheng $\mathrm{W}$. Histologic dating of the endometrium: accuracy, reproducibility, and practical value. Adv Anat Pathol. 2005;12(2):39-46.

19. Dal J, Vural B, Caliskan E, Ozkan S, Yucesoy I. Power Doppler ultrasound studies of ovarian, uterine, and endometrial blood flow in regularly menstruating women with respect to luteal phase defects. Fertil Steril. 2005;84(1):224-7.

20. Sterzik K, Abt M, Grab D, Schneider V, Strehler E. Predicting the histologic dating of an endometrial biopsy specimen with the use of Doppler ultrasonography and hormone measurements in patients undergoing spontaneous ovulatory cycles. Fertil Steril. 2000; 73(1):94-8.

21. Matijevic R, Grgic O. Predictive values of ultrasound monitoring of the menstrual cycle. Curr Opin Obstet Gynecol. 2005;17(4):405-10.

22. Tinkanen $\mathrm{H}$. The role of vascularisation of the corpus luteum in the short luteal phase studied by Doppler ultrasound. Acta Obstet Gynecol Scand. 1994;73(4):321-3.

23. Kurjak A, Kupesic-Urek S, Schulman H, Zalud I. Transvaginal color flow Doppler in the assessment of ovarian and uterine blood flow in fertile women. Fertil Steril 1991;56(5):870-3.

24. Webb R, Woad KJ, Armstrong DG. Corpus luteum (CL) function: local control mechanisms. Domest Anim Endocrinol. 2002;23(1-2):277-85.

25. Miyamoto A, Shirasuna K, Wijayagunawardane MP, Watanabe S, Hayashi M, Yamamoto D, et al. Blood flow: a key regulatory component of corpus luteum function in the cow. Domest Anim Endocrinol. 2005;29(2):329-39.

26. Malcolm CE, Cumming DC. Does anovulation exist in eumenorrheic women? Obstet Gynecol. 2003;102(2):317-8.

27.Evers JL. Female subfertility. Lancet. 2002;360(9327):151-9. 